\title{
Genomic organization and evolution of the ULBP genes in cattle Joshua H Larson ${ }^{1}$, Brandy M Marron ${ }^{2}$, Jonathan E Beever ${ }^{2}$, Bruce A Roe ${ }^{3}$ and Harris A Lewin*1
}

\begin{abstract}
Address: ${ }^{1}$ Laboratory of Immunogenetics, Department of Animal Sciences, University of Illinois at Urbana-Champaign, 210 Edward R. Madigan Laboratory, 1201 W. Gregory Dr., Urbana, IL 61801, USA, ²Laboratory of Molecular Genetics, Department of Animal Sciences, University of Illinois at Urbana-Champaign, 220 Edward R. Madigan Laboratory, 1201 W. Gregory Dr., Urbana, IL 61801, USA and ${ }^{3}$ Department of Chemistry and Biochemistry, Advanced Center for Genome Technology, University of Oklahoma, 2107 Stephenson Research and Technology Center, 101 David L. Boren Blvd, Norman, OK 73069, USA
\end{abstract}

Email: Joshua H Larson - jhlarson@uiuc.edu; Brandy M Marron - bhaton@uiuc.edu; Jonathan E Beever - jbeever@uiuc.edu; Bruce A Roe - broe@ou.edu; Harris A Lewin* - h-lewin@uiuc.edu

* Corresponding author

Published: 05 September 2006

BMC Genomics 2006, 7:227 doi:10.1/86/147|-2164-7-227
Received: 30 June 2006

Accepted: 05 September 2006

This article is available from: http://www.biomedcentral.com//47/-2/64/7/227

(c) 2006 Larson et al; licensee BioMed Central Ltd.

This is an Open Access article distributed under the terms of the Creative Commons Attribution License (http://creativecommons.org/licenses/by/2.0), which permits unrestricted use, distribution, and reproduction in any medium, provided the original work is properly cited.

\begin{abstract}
Background: The cattle ULI6-binding protein I (ULBPI) and ULBP2 genes encode members of the MHC Class I superfamily that have homology to the human ULBP genes. Human ULBPI and ULBP2 interact with the NKG2D receptor to activate effector cells in the immune system. The human cytomegalovirus ULI6 protein is known to disrupt the ULBP-NKG2D interaction, thereby subverting natural killer cell-mediated responses. Previous Southern blotting experiments identified evidence of increased ULBP copy number within the genomes of ruminant artiodactyls. On the basis of these observations we hypothesized that the cattle ULBPs evolved by duplication and sequence divergence to produce a sufficient number and diversity of ULBP molecules to deliver an immune activation signal in the presence of immunogenic peptides. Given the importance of the ULBPs in antiviral immunity in other species, our goal was to determine the copy number and genomic organization of the ULBP genes in the cattle genome.

Results: Sequencing of cattle bacterial artificial chromosome genomic inserts resulted in the identification of 30 cattle ULBP loci existing in two gene clusters. Evidence of extensive segmental duplication and approximately $14 \mathrm{Kbp}$ of novel repetitive sequences were identified within the major cluster. Ten ULBPs are predicted to be expressed at the cell surface. Substitution analysis revealed II outwardly directed residues in the predicted extracellular domains that show evidence of positive Darwinian selection. These positively selected residues have only one residue that overlaps with those proposed to interact with NKG2D, thus suggesting the interaction with molecules other than NKG2D.

Conclusion: The ULBP loci in the cattle genome apparently arose by gene duplication and subsequent sequence divergence. Substitution analysis of the ULBP proteins provided convincing evidence for positive selection on extracellular residues that may interact with peptide ligands. These results support our hypothesis that the cattle ULBPs evolved under adaptive diversifying selection to avoid interaction with a ULI6-like molecule whilst preserving the NKG2D binding site. The large number of ULBPs in cattle, their extensive diversification, and the high prevalence of bovine herpesvirus infections make this gene family a compelling target for studies of antiviral immunity.
\end{abstract}


Table I: BAC clone composition of the assembled ULBP gene clusters

\begin{tabular}{|c|c|c|c|c|c|}
\hline Assembled ULBP cluster (bp) & Component BAC clone (accession) & Size (bp) & Orient. & $\begin{array}{l}\text { Component } \mathrm{BAC} \text { sequence } \\
\text { regions used in assembly }\end{array}$ & $\begin{array}{l}\text { Corresponding ULBP } \\
\text { cluster region }\end{array}$ \\
\hline \multicolumn{6}{|l|}{ Minor ULBP cluster $(331,973)$} \\
\hline & 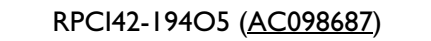 & 156,543 & + & $5-156,543$ & $1-156,539$ \\
\hline & $\mathrm{RPCl} 42-522 \mathrm{~F} 4$ (DQ405274) & 202,200 & + & $26,767-202,200$ & $|56,540-33|, 973$ \\
\hline \multicolumn{6}{|l|}{ Major ULBP cluster $(464,586)$} \\
\hline & CHORI240-2 IB24 (DQ405273) & 116,254 & + & $1-98,955$ & $1-98,955$ \\
\hline & 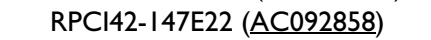 & 165,590 & - & $165,584-28,446$ & $98,956-236,094$ \\
\hline & 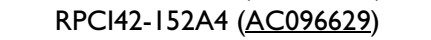 & 191,732 & - & $185,338-12 \mid, 525$ & $236,095-299,908$ \\
\hline & $\mathrm{RPCl} 42-146 \mathrm{CI}$ ( & 164,686 & + & $9-164,686$ & $299,909-464,586$ \\
\hline
\end{tabular}

\section{Background}

The cattle Major Histocompatibility Complex Class I-like Gene Family A (MHCLA) was initially discovered in a cattle spleen cDNA library during a search for highly divergent mammalian genes [1]. Two transcripts, MHCLA1 and MHCLA2, were found to be members of the MHC Class I superfamily, encoding cell-surface transmembrane proteins containing $\alpha 1$ - and $\alpha 2$-like domains, but no $\alpha 3$-like domain. These molecules have peptide sequence similarity to their homologues in other mammalian species, including the ULBP and RAET1 molecules in humans $[2,3]$ and the H60, RAE1 and MULT1 molecules in mice [4-7]. To establish consistency with the human nomenclature, the cattle MHCLA1 and MHCLA2 genes are renamed $U L B P 1$ and $U L B P 2$, respectively, in this study. The function of cattle ULBP molecules is not known, but the human and mouse homologues have been demonstrated to interact with the NKG2D receptor, leading to activation of natural killer (NK) cells and $\mathrm{T}$ cell subsets in antitumour and infectious disease immunity [8]. In vitro studies have demonstrated that the soluble human cytomegalovirus (hCMV) protein UL16 interferes with the ability of ULBP1 and ULBP2 to interact with NKG2D, and coexpression of UL16 with ULBP1 or ULBP2 results in cytoplasmic retention of the ULBP molecules $[2,9,10]$.

Southern blot analysis revealed the existence of a high copy number of ULBP genes in the cattle genome and seven other ruminant genomes. It was thus hypothesized that the cattle ULBP genes evolved rapidly by duplication and sequence divergence in response to selective pressure exerted by a viral pathogen(s). Extensive duplication of the cattle ULBP genes may serve to increase the repertoire of ULBP molecules able to bind NKG2D to initiate an immune response even in the presence of a UL16-like molecule [1].

The purpose of the present study was to identify the number of ULBP genes in cattle and describe their genomic organization. Six cattle bacterial artificial chromosome (BAC) clones were sequenced, resulting in the identification of 30 ULBP loci organized in two gene clus- ters on BTA9. Sequence analysis of the paralogues revealed that extensive gene duplication led to the present organization of the ULBP gene clusters. Bioinformatics tools were employed to characterize domains and sequence motifs in ten $U L B P$ genes predicted to encode cell surface molecules, the majority of which are predicted glycoproteins. Substitution analysis identified specific codons in these genes that appear to be under positive Darwinian selection, and these selected sites were interpreted in a structural context using homology modelling.

\section{Results \& discussion}

Identification of the minor and major ULBP gene clusters Four minimally overlapping ULBP-containing BACs were identified by hybridization-based screening with a fulllength cattle ULBP1 clone and then sequenced: RP42147E22 [GenBank: AC092858], RP42-152A4 [GenBank: AC096629], RP42-146C17 [GenBank: $\underline{\text { AC098686] and }}$ RP42-194O5 [GenBank: AC098687]. Sequence alignment revealed that the former three $\mathrm{BACs}$ were overlapping, and the latter BAC was a singleton. Using BAC-end sequence data, two additional minimally overlapping BAC clones were identified: RP42-522F4 [GenBank: DQ405274] and CHORI240-21B24 [GenBank: DQ405273]. The overlapping clones were used to reconstruct two gene clusters, termed the "minor" ULBP cluster [GenBank: DP000082], spanning 331,973 bp, and the "major" ULBP cluster [GenBank: DP000081], spanning 464,586 bp (Table 1). The minor and major cluster sequences could not be further extended or joined by querying publicly available cattle genome sequence data [NCBI Build 2.0]. The ULBP1 locus [1] was not identified in this study, and therefore the major ULBP cluster sequence may be incomplete upstream of $U L B P 7$.

Four ULBP loci were identified within the minor cluster, and $26 U L B P$ loci were identified in the major cluster. Nine loci represent coding sequences, and 21 loci are probable pseudogenes. Exons were identified by alignment and manual inspection (Table 2, 3). Loci were designated as genes if they contained uninterrupted coding sequence in the signal peptide, $\alpha 1$ and $\alpha 2$ domains. Loci 
Table 2: Genomic annotation of the minor ULBP gene cluster

\begin{tabular}{|c|c|c|c|c|c|c|}
\hline Locus & Status & Orient. & Exon & Exon position (bp) & Size (bp) & Stop codon \\
\hline \multirow[t]{13}{*}{ STXBP5 } & gene & + & 11 & $1,039-1,186$ & 148 & No \\
\hline & & & 12 & $1,390-1,456$ & 67 & No \\
\hline & & & 13 & $2,620-2,840$ & 221 & No \\
\hline & & & 14 & $3,330-3,508$ & 179 & No \\
\hline & & & 15 & $10,247-10,372$ & 126 & No \\
\hline & & & 16 & $|2,700-| 2,85 \mid$ & 152 & No \\
\hline & & & 17 & $18,793-18,858$ & 66 & No \\
\hline & & & 18 & $43,553-43,645$ & 93 & No \\
\hline & & & 19 & $44,900-45,269$ & 370 & No \\
\hline & & & 20 & $45,574-45,739$ & 166 & No \\
\hline & & & 21 & $53,349-53,460$ & 112 & No \\
\hline & & & 22 & $57,390-57,614$ & 225 & No \\
\hline & & & 23 & $59,839-60,297$ & 459 & Yes \\
\hline ULBP3 & pseudogene & + & 3 & $101,446-101,579$ & 134 & No \\
\hline \multirow{5}{*}{ ULBP4 } & gene & - & 1 & $|32,643-| 32,5 \mid 2$ & 132 & No \\
\hline & & & 2 & $124,69 \mid-124,428$ & 264 & No \\
\hline & & & 3 & $124,189-123,914$ & 276 & No \\
\hline & & & 4 & $123,310-123,175$ & 136 & No \\
\hline & & & 5 & $|2|, 996-|2|, 52 \mid$ & 476 & Yes \\
\hline ULBP5 & pseudogene & + & 2 & $137,458-137,605$ & 148 & No \\
\hline \multirow[t]{4}{*}{ ULBP6 } & & + & 2 & $154,996-155,258$ & 263 & Yes \\
\hline & & & 3 & $155,474-155,749$ & 276 & No \\
\hline & & & 4 & $156,396-156,527$ & 132 & Yes \\
\hline & & & 5 & $|57,7| \mid-157,835$ & 125 & Yes \\
\hline NFYB & gene & + & I & $\mid 84,327-185,342$ & 1,016 & Yes \\
\hline \multirow[t]{2}{*}{ SAMDCI } & gene & + & 1 & $230,021-230,485$ & 465 & No \\
\hline & & & 2 & $290,210-290,272$ & 63 & Yes \\
\hline
\end{tabular}

either lacking an exon corresponding to the signal peptide, $\alpha 1$ or $\alpha 2$ domains or containing a stop codon in the coding sequence of one of these three domains were designated as pseudogenes. Many of the pseudogenes contain exons with intact coding sequence (Table 2, 3). It may be speculated that these pseudogenes serve as a repository for generating novel ULBP paralogues through gene conversion.

The nine ULBP genes identified in this study have a canonical five exon structure. An exception is ULBP21, which has six exons; the first two exons encode the signal peptide. All nine ULBP genes contain GU/AG exon splicing motifs. Because of the high degree of interlocus sequence identity among ULBP genes (e.g., ULBP9 and ULBP27 have $99.8 \%$ nucleotide identity over $1252 \mathrm{bp}$ ), the assignment of expressed sequence tags (ESTs) to any particular locus was problematic. Thus, EST data could not be used to definitively support ULBP gene annotation.

\section{Comparative genome organization}

Both the cattle minor and major ULBP clusters were localized to BTA9 using radiation hybrid mapping methods (data not shown). Comparative analysis showed that STXBP5 and SAMDC1 share a conserved orientation on
HSA6q and BTA9 (Figure 1). The cattle ULBP3, ULBP4, ULBP5 and ULBPG loci located between STXBP5 and $S A M D C 1$ likely originated by duplication and insertion of genes from the major ULBP cluster (see below).

The cattle NFYB gene is orthologous to human NFYB on HSA12 (Figure 1). Comparison of nucleotide alignments in the cattle minor ULBP cluster and HSA12 genomic sequence demonstrates that sequence similarity is limited to the NFYB gene. The absence of genomic sequence similarity flanking this gene in humans and the lack of intronic sequence in cattle NFYB suggests that the cattle NFYB locus represents a retrotransposed gene. Although unlikely, a chimeric cattle BAC clone or sequence assembly error in the human genome cannot be ruled out as an explanation for these findings.

The discovery of at least 30 distinct cattle ULBP paralogues makes cattle the species with the largest number of ULBPlike genes identified to date (Figure 1). Our findings confirm and extend previous Southern blot analysis indicating a large number of $U L B P$ paralogues in cattle and seven other ruminant artiodactyl genomes [1]. In contrast, the more distantly related artiodactyls, swine and alpaca, appear to have relatively few ULBP genes $[1,11]$. 
Table 3: Genomic annotation of the major ULBP gene cluster

\begin{tabular}{|c|c|c|c|c|c|c|}
\hline Locus & Status & Orient. & Exon & Exon position (bp) & Size (bp) & Stop codon \\
\hline \multirow[t]{4}{*}{ ULBP7 } & pseudogene & - & 2 & $8,262-8,018$ & 245 & Yes \\
\hline & & & 3 & $7,790-7,515$ & 276 & No \\
\hline & & & 4 & $6,890-6,759$ & 132 & No \\
\hline & & & 5 & $5,578-5,454$ & 125 & Yes \\
\hline ULBP8 & pseudogene & - & 2 & $24,180-23,979$ & 202 & Yes \\
\hline \multirow[t]{5}{*}{ ULBP9 } & gene & + & 1 & $28,7|9-28,8| 3$ & 95 & No \\
\hline & & & 2 & $37,552-37,815$ & 264 & No \\
\hline & & & 3 & $38,054-38,329$ & 276 & No \\
\hline & & & 4 & $38,934-39,066$ & 133 & Yes \\
\hline & & & 5 & $39,881-40,391$ & 511 & Yes \\
\hline \multirow[t]{2}{*}{ ULBPIO } & pseudogene & - & 2 & $51,618-51,357$ & 262 & No \\
\hline & & & 3 & $51,115-50,835$ & 281 & Yes \\
\hline \multirow[t]{5}{*}{ ULBPII } & gene & + & I & $55,298-55,409$ & 112 & No \\
\hline & & & 2 & $64,155-64,418$ & 264 & No \\
\hline & & & 3 & $64,656-64,931$ & 276 & No \\
\hline & & & 4 & $65,535-65,667$ & 133 & No \\
\hline & & & 5 & $66,814-67,324$ & 511 & Yes \\
\hline \multirow[t]{2}{*}{ ULBPI 2} & pseudogene & - & 2 & $78,583-78,389$ & 195 & No \\
\hline & & & 3 & $78,079-77,816$ & 264 & No \\
\hline \multirow[t]{5}{*}{ ULBPI3 } & gene & + & 1 & $82,210-82,345$ & 136 & No \\
\hline & & & 2 & 91,064-91,327 & 264 & No \\
\hline & & & 3 & 91,564-91,839 & 276 & No \\
\hline & & & 4 & $92,443-92,578$ & 136 & No \\
\hline & & & 5 & $93,722-94,227$ & 506 & Yes \\
\hline \multirow[t]{2}{*}{ ULBP $\mid 4$} & pseudogene & - & 2 & $105,503-105,240$ & 264 & No \\
\hline & & & 3 & $105,005-104,733$ & 273 & Yes \\
\hline \multirow[t]{5}{*}{ ULBPI5 } & gene & + & 1 & $109,168-109,278$ & 111 & No \\
\hline & & & 2 & $117,844-118,107$ & 264 & No \\
\hline & & & 3 & $118,345-118,620$ & 276 & No \\
\hline & & & 4 & $119,224-119,356$ & 133 & No \\
\hline & & & 5 & $|20,500-12|, 003$ & 504 & Yes \\
\hline \multirow[t]{2}{*}{ ULBPI 6} & pseudogene & - & 2 & $|4|, 435-14|| 60$, & 276 & No \\
\hline & & & 3 & $|40,93|-\mid 40,666$ & 266 & No \\
\hline \multirow[t]{5}{*}{ ULBPI 7} & gene & + & I & $|60,588-160,72|$ & 134 & No \\
\hline & & & 2 & $178,284-178,547$ & 264 & No \\
\hline & & & 3 & $178,782-179,057$ & 276 & No \\
\hline & & & 4 & $179,685-179,817$ & 133 & No \\
\hline & & & 5 & $|8|,|95-181,59|$ & 397 & Yes \\
\hline \multirow[t]{2}{*}{ ULBPI8 } & pseudogene & - & 2 & $194,201-194,003$ & 199 & No \\
\hline & & & 3 & $192,969-192,694$ & 276 & Yes \\
\hline \multirow[t]{3}{*}{ ULBPI9 } & pseudogene & - & 2 & $205,757-205,495$ & 263 & No \\
\hline & & & 3 & $205,259-204,985$ & 275 & Yes \\
\hline & & & 4 & $204,053-203,923$ & $13 \mid$ & Yes \\
\hline ULBP20 & pseudogene & - & 3 & $215,364-215,116$ & 249 & Yes \\
\hline \multirow[t]{6}{*}{ ULBP2 I } & gene & - & 1 & $230,458-230,37 \mid$ & 88 & No \\
\hline & & & 2 & $227,628-227,590$ & 39 & No \\
\hline & & & 3 & $226,923-226,660$ & 264 & No \\
\hline & & & 4 & $226,284-226,009$ & 276 & No \\
\hline & & & 5 & $224,903-224,77 \mid$ & 133 & Yes \\
\hline & & & 6 & $223,621-223,508$ & 114 & Yes \\
\hline \multirow[t]{4}{*}{ ULBP22 } & pseudogene & - & 2 & $247,327-247,005$ & 263 & Yes \\
\hline & & & 3 & $246,855-246,580$ & 276 & No \\
\hline & & & 4 & $245,961-245,830$ & 132 & No \\
\hline & & & 5 & $244,644-244,532$ & 113 & No \\
\hline ULBP23 & pseudogene & - & 2 & $264,695-264,431$ & 264 & Yes \\
\hline \multirow[t]{4}{*}{ ULBP24 } & pseudogene & + & 1 & $270,030-270,163$ & 134 & No \\
\hline & & & 2 & $277,94 I-278,215$ & 275 & Yes \\
\hline & & & 3 & $278,442-278,7 \mid 7$ & 276 & No \\
\hline & & & 4 & $279,606-279,738$ & 133 & Yes \\
\hline
\end{tabular}


Table 3: Genomic annotation of the major ULBP gene cluster (Continued)

\begin{tabular}{|c|c|c|c|c|c|c|}
\hline & & & 5 & $280,568-281,054$ & 487 & Yes \\
\hline \multirow[t]{6}{*}{ ULBP25 } & pseudogene & - & 1 & $300,310-300,223$ & 88 & No \\
\hline & & & 2 & $296,899-296,861$ & 39 & No \\
\hline & & & 3 & $296,|94-295,93|$ & 264 & No \\
\hline & & & 4 & $295,553-295,279$ & 275 & Yes \\
\hline & & & 5 & $294,125-294,015$ & 111 & Yes \\
\hline & & & 6 & $292,846-292,384$ & 463 & Yes \\
\hline \multirow[t]{5}{*}{ ULBP26 } & pseudogene & - & I & $322,695-322,562$ & 134 & No \\
\hline & & & 2 & $316,336-316,098$ & 239 & Yes \\
\hline & & & 3 & $315,865-315,589$ & 277 & Yes \\
\hline & & & 4 & $3|4,950-3| 4,8 \mid 5$ & 136 & No \\
\hline & & & 5 & $3|4|,|3-3| 3,670$ & 444 & Yes \\
\hline \multirow[t]{5}{*}{ ULBP27 } & gene & + & I & $336,705-336,840$ & 134 & No \\
\hline & & & 2 & $345,589-345,852$ & 264 & No \\
\hline & & & 3 & $346,091-346,366$ & 276 & No \\
\hline & & & 4 & $346,97 \mid-347,103$ & 133 & Yes \\
\hline & & & 5 & $347,918-348,404$ & 487 & Yes \\
\hline \multirow[t]{3}{*}{ ULBP28 } & pseudogene & - & 2 & $359,653-359,390$ & 264 & No \\
\hline & & & 3 & $359,148-358,883$ & 266 & No \\
\hline & & & 4 & $358,026-357,887$ & 140 & Yes \\
\hline \multirow[t]{5}{*}{ ULBP2 } & gene & + & 1 & $378,496-378,629$ & 134 & No \\
\hline & & & 2 & $386,342-386,605$ & 264 & No \\
\hline & & & 3 & $386,840-387,115$ & 276 & No \\
\hline & & & 4 & $387,763-387,895$ & 133 & No \\
\hline & & & 5 & $389,276-389,762$ & 487 & Yes \\
\hline \multirow[t]{2}{*}{ ULBP29 } & pseudogene & - & 2 & $402,249-402,066$ & 184 & No \\
\hline & & & 3 & $40|, 0| 8-400,743$ & 276 & Yes \\
\hline \multirow[t]{3}{*}{ ULBP30 } & pseudogene & - & 2 & $4|3,8||-4| 3,548$ & 264 & No \\
\hline & & & 3 & $4|3,3| 3-4 \mid 3,038$ & 276 & No \\
\hline & & & 4 & $4|2||| 2-,4||, 980$ & 133 & Yes \\
\hline ULBP3I & pseudogene & - & 3 & $423,435-423,164$ & 272 & Yes \\
\hline
\end{tabular}

The cattle ULBP loci evolved through extensive gene duplication

The cattle minor and major ULBP clusters were analyzed for internal nucleotide sequence similarity (Figure 2 and Figure 3, respectively) in order to identify duplicated segments. The largest was a duplication of ULBP28, ULBP2, ULBP29, ULBP30, and ULBP31 to form ULBP16, ULBP17, ULBP18, ULBP19, and ULBP20 (Figure 3). The directionality of this duplication event was determined from the expansion of a novel cattle-specific repeat (see below) in the first intron of cattle ULBP17 as compared to the smaller corresponding repeat region in the first intron of ULBP2. There appear to be four tandem duplications involving blocks containing ULBP9 and ULBP10, ULBP11 and ULBP12, ULBP13 and ULBP14, and ULBP15 (Figure $3)$. However, similarity to ULBP27 was observed for ULBP9, ULBP11, ULBP13, and ULBP15, thus providing evidence that $U L B P 27$ was likely also part of the large duplication involving ULBP28 through ULBP31 described above. In addition to the duplication events described above, there are two other segments that contain duplicated genes: i) $U L B P 7, U L B P 8$, and $U L B P 9$ are related to ULBP22, ULBP23, and ULBP24, and ii) ULBP21 and ULBP22 are related to ULBP25 and ULBP26 (Figure 3).
Known repetitive elements were identified in the minor and major ULBP clusters (Table 4). An additional novel genomic repeat was identified within the first introns of ULBP17 and ULBP2. The novel repeat spans $11,938 \mathrm{bp}$ in the first intron of $U L B P 17$ and 2,100 bp in the first intron of ULBP2 (Figure 3) [GenBank: DP000081]. These repeats are specific to the cattle major ULBP cluster and are not found elsewhere in the cattle genome. The large size of the $U L B P 17$ repeat region relative to the corresponding repeat region in ULBP2 suggests active repeat expansion. A full understanding of the means by which these repeats contributed to the evolution of the ULBP gene family awaits complete genomic sequencing of this region and sequencing of additional haplotypes.

\section{Structure and evolution of ULBP proteins}

Conceptual translations of the nine cattle ULBP genes identified in this study and the previously identified cattle ULBP1 [1] are shown in Figure 4. Each molecule contains a 24 to 42 amino acid (aa) signal peptide sequence, an 88 aa $\alpha 1$ domain, an 84 aa $\alpha 2$ domain and a 25 to 30 aa connecting peptide region followed by a hydrophobic segment. Peptide sequence identity was determined within the $\alpha 1$ and $\alpha 2$ domains for each cattle ULBP and the por- 


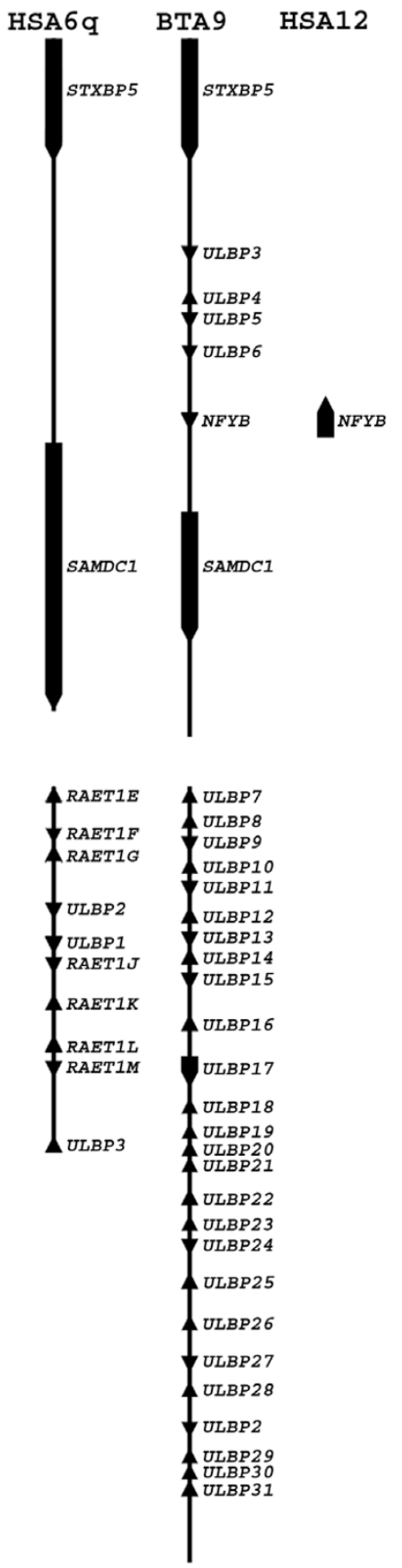

Figure I

Scaled comparative map showing homologous ULBP. containing chromosome regions in cattle and human. Arrows indicate gene orientation. The BTA9 upper and lower seqments represent the major and minor ULBP clusters, respectively. Cattle BAC contig sizes and gene positions are found in Tables I-3. The upper and lower chromosomal segments from HSA6q depict sequence positions 147,566$148,013 \mathrm{Kbp}$ and 150,302-I50,482 Kbp [NCBI Build 35], respectively. The HSAI 2 chromosomal segment represents positions 103,013-103,034 Kbp [NCBI Build 35].
Table 4: Repetitive element composition of the minor and major ULBP gene clusters

\begin{tabular}{|c|c|c|}
\hline Repetitive element & Minor ULBP cluster & Major ULBP cluster \\
\hline SINE & 5,681 bp $(10.1 \%)$ & 37,368 bp (8.8\%) \\
\hline LINE & 9,973 bp (17.7\%) & 69,177 bp (16.3\%) \\
\hline LTR & 2,406 bp (4.3\%) & $18,16 \mid$ bp $(4.3 \%)$ \\
\hline DNA (including MERI/2) & 2,388 bp (4.2\%) & 14,749 bp (3.5\%) \\
\hline Small RNA & 0 bp $(0.0 \%)$ & 219 bp (0.1\%) \\
\hline Satellites & 0 bp (0.0\%) & 0 bp $(0.0 \%)$ \\
\hline Simple repeats & 893 bp (I.6\%) & 2,446 bp $(0.6 \%)$ \\
\hline Low complexity & 394 bp (0.7\%) & 1,614 bp $(0.4 \%)$ \\
\hline Total repetitive elements & 21,735 bp (38.6\%) & 143,734 bp (34.0\%) \\
\hline
\end{tabular}

Repetitive element statistics were generated only from genomic regions flanked by ULBP loci. These include 56,390 bp of the minor cluster and $423,435 \mathrm{bp}$ of the major cluster.

cine ULBP (Figure 5) [11]. ULBP9, ULBP21, and ULBP27 have glycophosphatidylinositol (GPI) anchor sites (P < $0.01, \mathrm{P}<0.05$, and $\mathrm{P}<0.001$, respectively). The other seven ULBPs have predicted transmembrane domains of 23 to 25 aa followed by cytoplasmic tails ranging from 27 to 73 aa in length. The signal sequences and transmembrane domain or GPI anchor motifs indicate that all 10 of the expressed ULBPs are localized extracellularly. Each protein has predicted $\mathrm{N}$-glycosylation motifs, with the exceptions of ULBP2 and ULBP17, suggesting that at least eight cattle ULBPs are glycoproteins.

Pairwise substitution analyses of ten ULBP genes showed an average global nonsynonymous to synonymous substitution ratio $\left(\omega_{t}\right)$ of 0.934 (Figure 6). Values of $\omega>1.0$ are regarded as indicating that positive selection has operated on the sequences analyzed [12]; however, global substitution analysis is stringent and may mask evidence of positive selection in molecular subregions [13]. Heterogeneity in selection intensity was investigated within the ULBP $\alpha 1$ and $\alpha 2$ domain regions (Table 5). In model 2 (M2), a continuous positive selection model with an additional (third) ratio of nonsynonymous to synonymous substitutions $\left(\omega_{2}\right)$ estimated from the data, $\omega_{2}$ is 3.17 , but represented only a small proportion $\left(\mathrm{p}_{2}=0.08\right.$ out of 1.0$)$ of codon sites. The log likelihood test of M2 vs. M1, the continuous neutrality model, was not statistically significant. In $\mathrm{M} 3$, the unconstrained discrete positive selection model, $\omega_{2}$ is 1.90 with $p_{2}=0.28$. The log likelihood test of M3 vs M1 was significant, providing evidence of heterogeneity in $\omega$ ratios among codon sites. Model 8, a beta distribution with an added $\omega$ class estimated from the data, was compared to $M 7$, a beta distribution that did not allow for positively selected sites. The log likelihood test of M8 vs M7 was significant, allowing the detection of positively selected codon sites (Table 5). Thirteen codon sites were determined to be under a high degree of positive selection (>90\% probability, Figure 4, Table 5). 


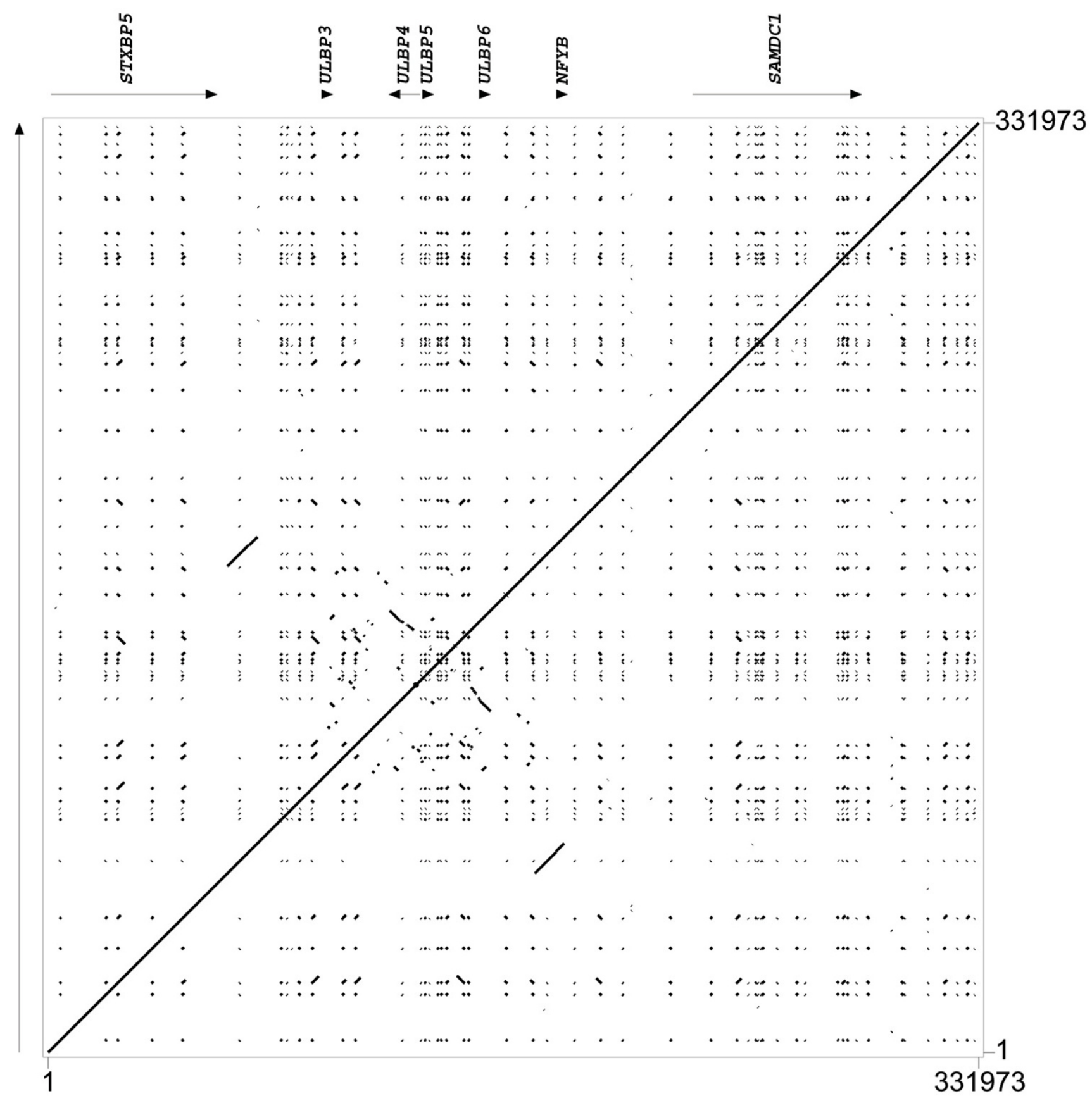

Figure 2

Internal sequence identity plot of the minor ULBP cluster. Sequence numbers displayed on the $X$ - and $Y$-axes indicate alignment orientation, originating in the lower left corner. The central diagonal line represents identity; other lines indicate regions of internal sequence identity. Genes are annotated above the figure with arrows indicating orientation. Nucleotide sequences used to construct the minor ULBP cluster are listed in Table I.

Twelve of the positively selected sites in the cattle ULBPs were mapped onto the three dimensional structure of human ULBP3 [PDB: 1KCG, chain C] (Figure 7). Eleven of the twelve positively selected residues were located at outwardly directed positions, indicating that positive selection acted at the level of interaction between the ULBPs and another molecule. On the basis of the structural data, fourteen human ULBP3 sites interact with 


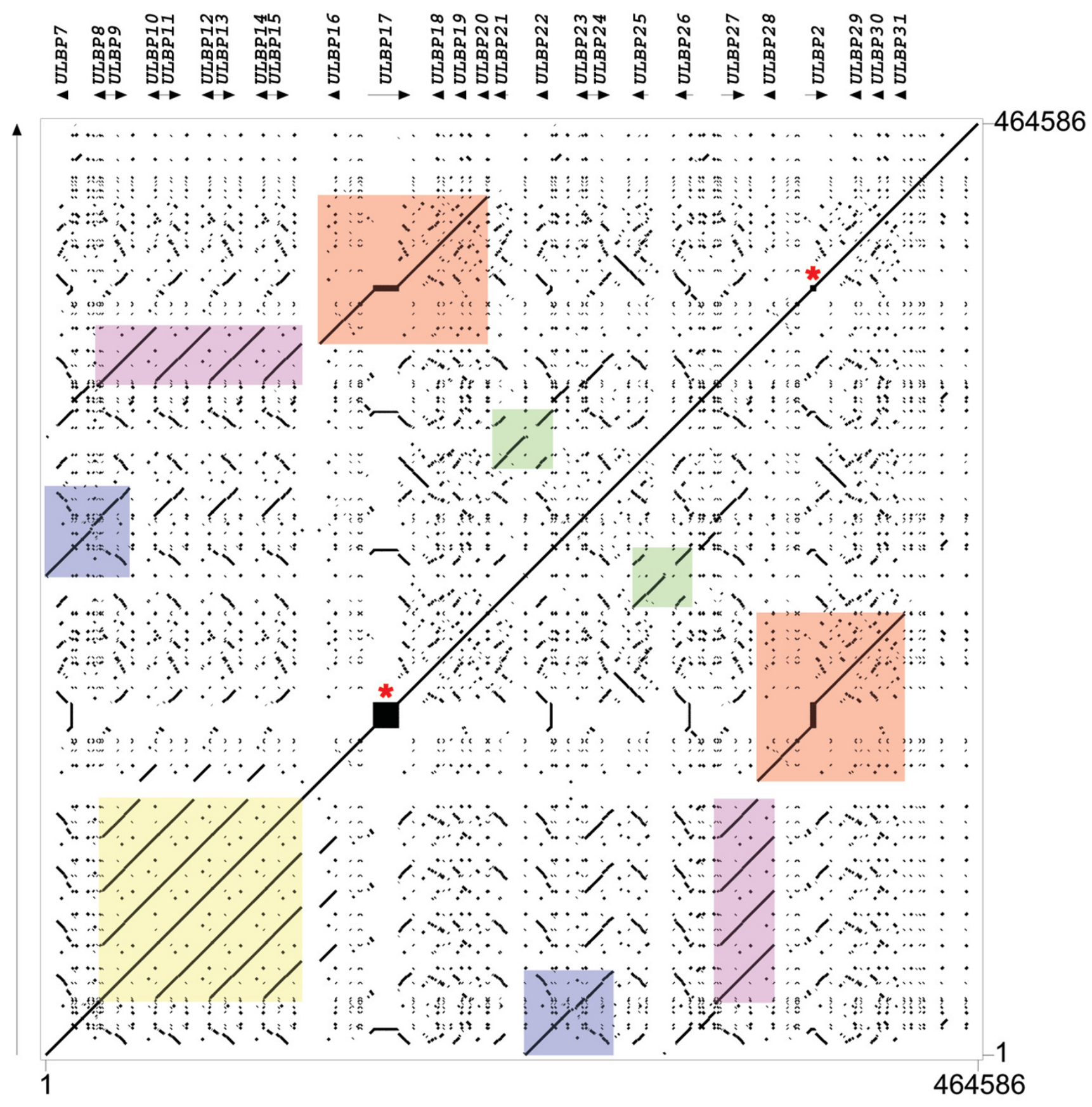

Figure 3

Internal sequence identity plot of the major ULBP cluster. Sequence numbers displayed on the $X$ - and $Y$-axes indicate alignment orientation, originating in the lower left corner. The center line represents identity; other lines indicate regions of internal sequence identity. Genes are annotated above the figure with arrows indicating orientation. Nucleotide sequences used to construct the major ULBP cluster are listed in Table I. The duplication of ULBP28-ULBP3 I to form ULBPI6-ULBP20 is within the red shaded area. The cattle specific repeat regions within ULBPI 7 and ULBP2 are indicated by red asterisks. The duplication of ULBP7-ULBP9 corresponding to ULBP22-ULBP24 is within the blue shaded area. The duplication of ULBP2IULBP22 corresponding to ULBP25-ULBP26 is within the green shaded area. The tandem duplications of ULBP9 and ULBPI0, ULBPI I and ULBPI 2, ULBPI 3 and ULBPI4, and ULBPI5 are within the yellow shaded area, and lines showing their similarity to the ULBP27 region are within the violet shaded area. 


\begin{tabular}{|c|c|c|c|c|c|c|c|c|c|}
\hline & ULBP2ULBP4ULE & P11U & LBP & ULBP21 & ULBP27 & PULBP & & & \\
\hline ULBP1 & $63 \quad 93$ & 92 & 96 & 85 & 94 & 63 & 64 & 93 & 52 \\
\hline ULBP2 & 62 & 62 & 63 & 60 & 64 & 98 & 59 & 62 & 50 \\
\hline ULBP4 & & 96 & 96 & 80 & 89 & 62 & 62 & 97 & 53 \\
\hline ULBP9 & & & 95 & 80 & 88 & 62 & 62 & 98 & 51 \\
\hline ULBP11 & & & & 84 & 93 & 63 & 64 & 96 & 52 \\
\hline ULBP13 & & & & & 81 & 59 & 59 & 80 & 47 \\
\hline ULBP15 & & & & & & 64 & 65 & 89 & 55 \\
\hline ULBP17 & & & & & & & 59 & 62 & 49 \\
\hline ULBP21 & & & & & & & & 64 & 62 \\
\hline \multirow[t]{2}{*}{ ULBP27 } & & & & & & & & & 52 \\
\hline & ULBP2ULBP4ULE & P11U & LBP & ULBP21 & ULBP27 & PULBP & & & \\
\hline ULBP1 & 65 & 86 & 91 & 87 & 85 & 67 & 70 & 86 & 55 \\
\hline ULBP2 & 67 & 63 & 65 & 61 & 61 & 88 & 71 & 63 & 54 \\
\hline ULBP4 & & 84 & 90 & 85 & 82 & 65 & 69 & 84 & 54 \\
\hline ULBP9 & & & 89 & 79 & 81 & 65 & 68 & 100 & 56 \\
\hline ULBP11 & & & & 85 & 86 & 69 & 74 & 89 & 57 \\
\hline ULBP13 & & & & & 85 & 62 & 65 & 79 & 48 \\
\hline ULBP15 & & & & & & 62 & 68 & 81 & 56 \\
\hline ULBP17 & & & & & & & 76 & 65 & 57 \\
\hline ULBP21 & & & & & & & & 68 & 55 \\
\hline ULBP27 & & & & & & & & & 56 \\
\hline
\end{tabular}

\section{Figure 5}

Sequence identity in the extracellular domains ofcattle and swine ULBPs. Percent pair-wise sequence identity between the $\alpha I$ and $\alpha 2$ domains of ten cattle ULBP proteins and porcine ULBP [GenBank: AAP8|932]. Sequence alignments were made using BLASTP and edited manually.

NKG2D [14], and only one of these binding residues was found to overlap with the cattle ULBP sites under positive selection (Figure 7). Therefore, the positively selected cattle ULBP sites, located outside of the predicted NKG2Dbinding residues on the basis of the homology modelling data, appear to interact with molecules other than NKG2D.

Several members of the Herpesviridae, which is the taxonomic family to which hCMV belongs, infect cattle, including bovine herpesviruses- 1 through -5 and bovine lymphotrophic herpesvirus. The sequenced genomes of bovine herpesvirus- $1,-4$, and -5 do not encode molecules with detectable sequence similarity to UL16 of hCMV
(HHV5); however it is conceivable that peptides encoded by the bovine herpesviruses or other viral pathogens may disrupt ULBP cell surface expression or the molecular interactions mediated by ULBP molecules. Thus, the rapid expansion of the ULBP gene family and the maintenance of such a large gene cluster are likely adaptive, serving to provide cattle with at least ten ULBP molecules through which an immune activation signal can be transmitted, even in the presence of an inhibitory pathogen-derived peptide.

\section{Conclusion}

This study provides insights into the genomic organization and evolution of the cattle ULBP genes, a recently

Table 5: Likelihood ratio tests of $\omega$ variation and identification of molecular sites under positive selection in ten cattle ULBP proteins

\begin{tabular}{ccccl}
\hline$-2(\mathrm{M} 2$ vs $\mathrm{MI})$ & $-2(\mathrm{M} 3$ vs MI $)$ & $-2(\mathrm{M} 8$ vs M7) & Parameter estimates for $M 8$ & Positively selected sites \\
\hline $3.2(P<0.25)$ & $10.6(P<0.05)$ & $8.1(P<0.025)$ & $P_{1}=0.279, P_{0}=0.721 \omega=1.904, \beta(60.6,99.0)$ & $\begin{array}{l}\mathbf{6 4}, \mathbf{6 8}, 69,70,99,106,144,165,178,190, \\
192,198,206\end{array}$
\end{tabular}

Accession numbers associated with the sequences analyzed are listed in the Methods section. MI, M2, M3, M7 and M8 refer to maximum likelihood models of $\omega$ ratios, and $-2(M 2$ vs $M I),-2(M 3$ vs $M I)$ and $-2(M 8$ vs $M 7)$ indicate the negative of two times the log likelihood difference between the selection and neutral models compared. $\mathrm{P}$ values for the test statistics are shown in parentheses. For $M 8, \mathrm{P}_{1}$ is the proportion of positively selected sites, $p_{0}$ is the proportion of sites not under positive selection, $\omega$ is the $d_{N} / d_{S}$ ratio for the selected sites, and $\beta(p$, $q)$ describes the beta distribution function. Positively selected ULBP sites are presented according to their numbered positions in the ULBPI preprotein sequence. Posterior probabilities for positively selected sites are represented in normal text (probability $>0.90$ ), bold text (probability $>0.95$ ), and italicized bold text (probability > 0.99). 


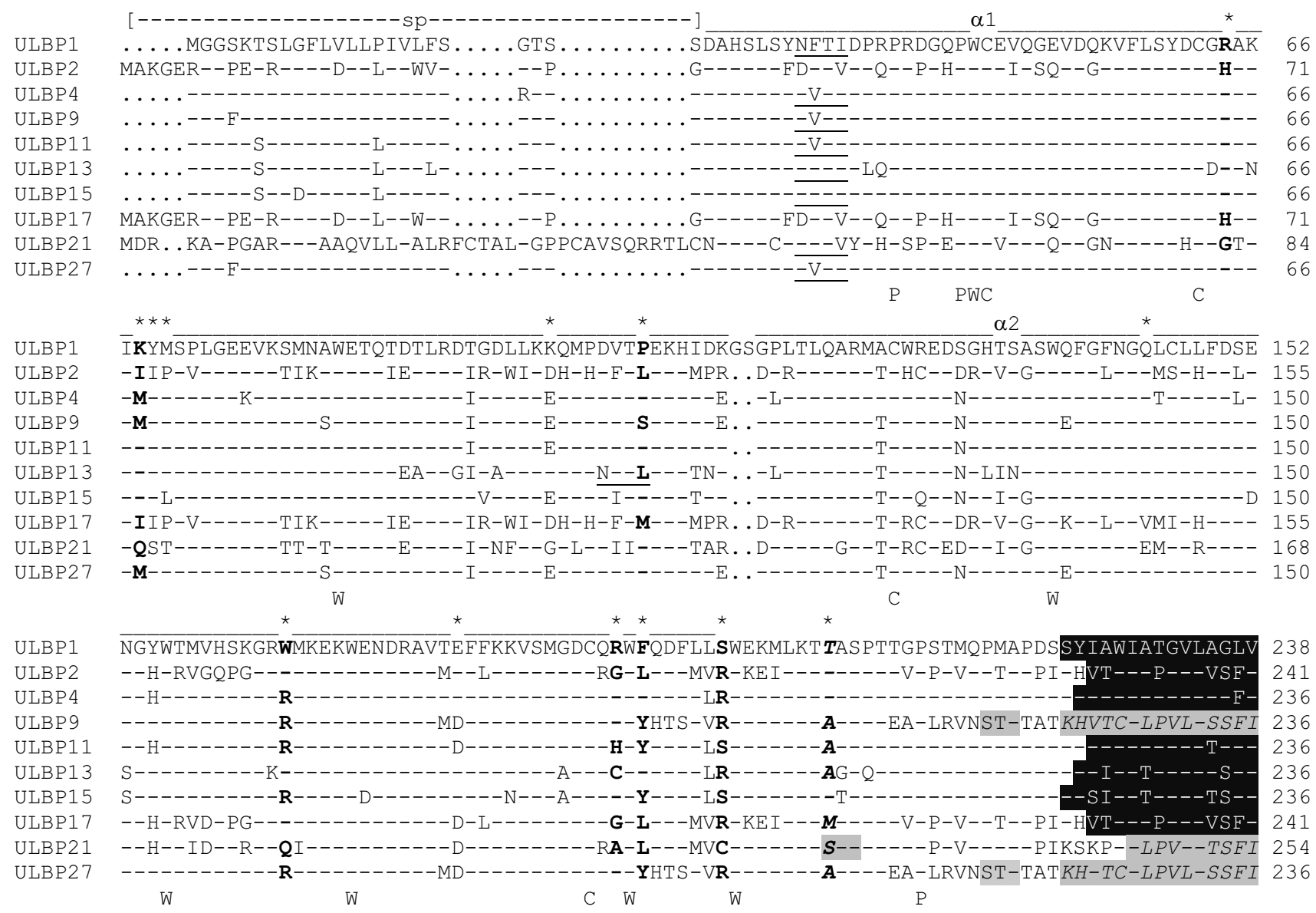

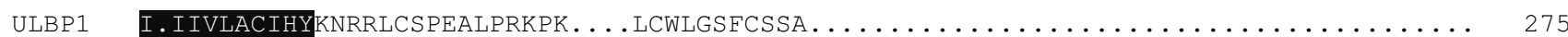

ULBP2 -KG--.-W-L--K-----Q--PD-CSVGLRTQSL--C---P-FTLEPRDQTLGVSSLSTSYDDTVAAPSRVSCQI . . . . . 315

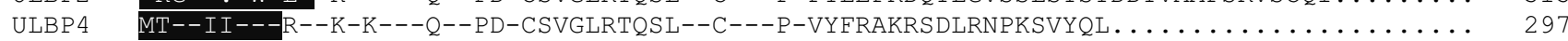

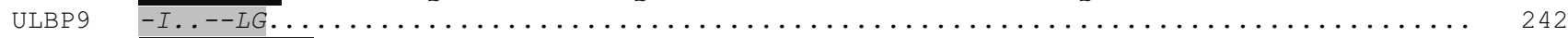

ULBP11

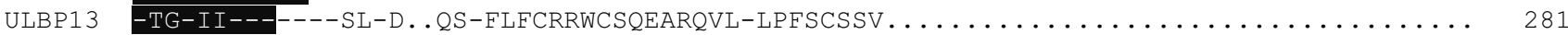

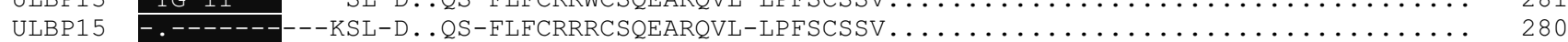

ULBP17 -MG--.-W-L--K-----Q--PD-CSVGLRTQ-L--Y---P-FTLEPRDQTLGVSSLSTSYDDTVAAPSRVSCQI . . . . . 315

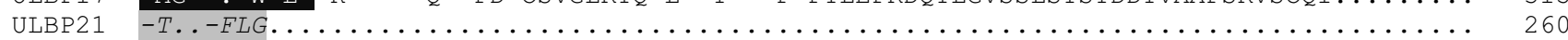

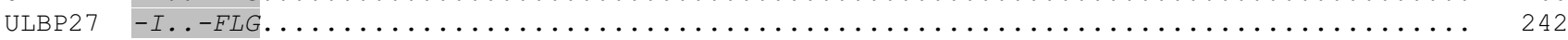

\section{Figure 4}

Protein alignment of the cattle ULBP family. Alignment gaps are represented by periods, and aligned ULBP sites sharing identity with cattle ULBPI are represented by dashes. The signal peptide region (sp) is represented by dashes within brackets above the alignment. The $\alpha \mathrm{I}$ and $\alpha 2$ domains are designated by an underscore above the alignment. Universally conserved cysteine, proline, and tryptophan residues are annotated with Cs, Ps and Ws, respectively, beneath the alignment. Predicted Nglycosylation motifs are represented by underlined text in the alignment. Transmembrane domains are represented by black shaded background. Predicted GPI anchor motifs are represented by gray shaded background, and the associated downstream hydrophobic regions are indicated by italicized text with gray shaded background. Positively selected sites are indicated by asterisks above the alignment, and posterior probabilities associated with each positively selected site (see Table 2.5) are represented within the alignment by normal text (probability $>0.90)$, bold text (probability $>0.95$ ), and italicized bold text (probability $>0.99)$.

expanded MHC class I-like gene family in cattle with a probable role in antiviral immunity. For the first time, evidence of positive Darwinian selection on non-NKGD2- binding residues was obtained, strongly implicating immunogenic peptides as the driving force of molecular evolution of the cattle ULBPs. The stage is now set for 


\begin{tabular}{|c|c|c|c|c|c|c|c|c|c|}
\hline & ULBP2 & ULBP 4 & ULBP 9 & ULBP 11 & ULBP 13 & $U L B P 15$ & ULBP 17 & $U L B P 21$ & ULBP 27 \\
\hline$U L B P 1$ & $\begin{array}{c}0.804 \\
(0.226 / \\
0.281)\end{array}$ & $\begin{array}{c}0.766 \\
(0.035 / \\
0.046)\end{array}$ & $\begin{array}{c}0.992 \\
(0.054 / \\
0.054)\end{array}$ & $\begin{array}{c}0.354 \\
(0.028 / \\
0.079)\end{array}$ & $\begin{array}{l}1.713 \\
(0.065 / \\
0.038)\end{array}$ & $\begin{array}{c}1.062 \\
(0.045 / \\
0.042)\end{array}$ & $\begin{array}{c}0.777 \\
(0.230 / \\
0.297)\end{array}$ & $\begin{array}{c}0.616 \\
(0.187 / \\
0.303)\end{array}$ & $\begin{array}{c}0.943 \\
(0.051 / \\
0.054)\end{array}$ \\
\hline ULBP2 & & $\begin{array}{c}0.819 \\
(0.222 / \\
0.271)\end{array}$ & $\begin{array}{c}0.878 \\
(0.244 / \\
0.278)\end{array}$ & $\begin{array}{c}0.741 \\
(0.230 / \\
0.311)\end{array}$ & $\begin{array}{c}0.786 \\
(0.250 / \\
0.318)\end{array}$ & $\begin{array}{c}0.927 \\
(0.242 / \\
0.261)\end{array}$ & $\begin{array}{l}1.272 \\
(0.034 / \\
0.027)\end{array}$ & $\begin{array}{c}0.731 \\
(0.208 / \\
0.284)\end{array}$ & $\begin{array}{c}0.891 \\
(0.242 / \\
0.272)\end{array}$ \\
\hline ULBP 4 & & & $\begin{array}{c}0.565 \\
(0.049 / \\
0.086)\end{array}$ & $\begin{array}{c}0.733 \\
(0.033 / \\
0.045)\end{array}$ & $\begin{array}{l}3.680 \\
(0.082 / \\
0.022)\end{array}$ & $\begin{array}{c}2.171 \\
(0.065 / \\
0.030)\end{array}$ & $\begin{array}{c}0.749 \\
(0.233 / \\
0.311)\end{array}$ & $\begin{array}{c}0.597 \\
(0.192 / \\
0.321)\end{array}$ & $\begin{array}{c}0.534 \\
(0.046 / \\
0.086)\end{array}$ \\
\hline ULBP 9 & & & & $\begin{array}{c}0.296 \\
(0.036 / \\
0.120)\end{array}$ & $\begin{array}{c}1.159 \\
(0.094 / \\
0.081)\end{array}$ & $\begin{array}{c}0.883 \\
(0.076 / \\
0.086)\end{array}$ & $\begin{array}{c}0.834 \\
(0.246 / \\
0.294)\end{array}$ & $\begin{array}{c}0.577 \\
(0.203 / \\
0.352)\end{array}$ & $\begin{array}{c}\text { NA } \\
(0.0025 / \\
0.000)\end{array}$ \\
\hline ULBP 11 & & & & & $\begin{array}{c}1.404 \\
(0.074 / \\
0.052)\end{array}$ & $\begin{array}{c}0.766 \\
(0.046 / \\
0.060)\end{array}$ & $\begin{array}{c}0.656 \\
(0.230 / \\
0.350)\end{array}$ & $\begin{array}{c}0.492 \\
(0.172 / \\
0.349)\end{array}$ & $\begin{array}{c}0.275 \\
(0.033 / \\
0.120)\end{array}$ \\
\hline ULBP 13 & & & & & & $\begin{array}{c}2.666 \\
(0.079 / \\
0.030)\end{array}$ & $\begin{array}{c}0.761 \\
(0.255 / \\
0.335)\end{array}$ & $\begin{array}{c}0.612 \\
(0.214 / \\
0.350)\end{array}$ & $\begin{array}{l}1.203 \\
(0.093 / \\
0.077)\end{array}$ \\
\hline ULBP 15 & & & & & & & $\begin{array}{c}0.832 \\
(0.245 / \\
0.294)\end{array}$ & $\begin{array}{c}0.658 \\
(0.190 / \\
0.288)\end{array}$ & $\begin{array}{c}0.851 \\
(0.073 / \\
0.086)\end{array}$ \\
\hline ULBP 17 & & & & & & & & $\begin{array}{c}0.672 \\
(0.203 / \\
0.303)\end{array}$ & $\begin{array}{c}0.835 \\
(0.246 / \\
0.294)\end{array}$ \\
\hline ULBP21 & & & & & & & & & $\begin{array}{c}0.568 \\
(0.200 / \\
0.352)\end{array}$ \\
\hline
\end{tabular}

Figure 6

Global substitution analysis of ten ULBP paralogues. The bold numerical values correspond to $\omega_{t}$ ratios; raw $d_{N} / d_{s} v a l-$ ues are listed in parentheses. Accession numbers associated with each sequence analyzed are listed in the Methods.

studying the role ULBPs play in cattle immunity during infection by viral pathogens, as well as their organization and evolution in other mammals.

\section{Methods}

\section{BAC selection, isolation and sequencing}

To identify BAC clones containing ULBP genes, filter membranes containing the RPCI-42 male Holstein BAC library (12X genome coverage; Children's Hospital Oakland Research Institute) were screened by Southern blot hybridization using the full-length ULBP1 [GenBank: AF317556] cDNA clone as a probe. Probe amplification and labelling, membrane hybridization, washing conditions and autoradiography were performed as previously described [1]. ULBP-containing BACs were cultured in 3 $\mathrm{ml} 2 \mathrm{x} \mathrm{LB}$ media with $20 \mu \mathrm{g} / \mathrm{ml}$ chloramphenicol (Sigma) overnight at $37^{\circ} \mathrm{C}$ with shaking. Cultures were centrifuged at $3000 \times g$ for $3 \mathrm{~min}$. The cell pellet was resuspended in $400 \mu \mathrm{l}$ of a solution containing $0.05 \mathrm{M}$ Tris, $0.01 \mathrm{M}$ EDTA ( $\mathrm{pH} 7.5$ ) and $50 \mu \mathrm{g} / \mathrm{ml}$ RNase A (Sigma), lysed by addition of $400 \mu \mathrm{l}$ of a solution containing $0.2 \mathrm{~N} \mathrm{NaOH}$ and $1 \%$ SDS, neutralized by addition of $400 \mu \mathrm{l}$ of a solution containing $4 \mathrm{M}$ guanidine- $\mathrm{HCl}$ and $0.75 \mathrm{M} \mathrm{KOAc}(\mathrm{pH}$ 4.6), and centrifuged at $10,000 \times g$ for $10 \mathrm{~min}$. An $860 \mu \mathrm{l}$ aliquot of cleared lysate was combined with $600 \mu \mathrm{l}$ isopropanol, placed on ice for $15 \mathrm{~min}$, and centrifuged at $10,000 \times g$ for $5 \mathrm{~min}$. The supernatant was decanted, and the pellet was washed with $500 \mu \mathrm{l} 70 \%$ ethanol before 


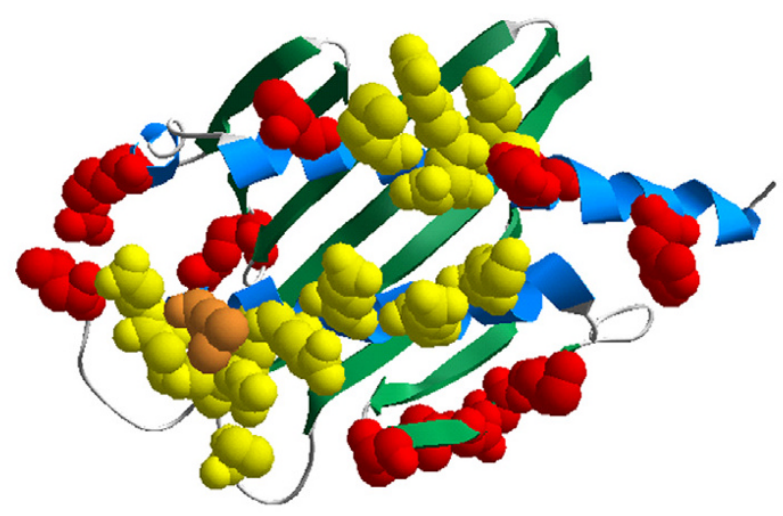

Figure 7

Positively selected ULBP sites mapped onto the crystal structure of human ULBP3. Positively selected cattle ULBP sites mapped onto the structure of human ULBP3.

Tertiary structure of human ULBP3 [PDB: IKCG, chain C] showing the spatial arrangement of homologous cattle ULBP residues under positive selection ( $>90 \%$ probability) as well as the human ULBP3 residues that interact with the NKG2D molecule. The human ULBP3 backbone appears in blue and green. Twelve of I 3 cattle ULBP sites under positive selection are mapped onto the structure, and eleven appear as red space-filling residues. Thirteen of the fourteen human ULBP3 sites that interact with NKG2D appear as yellow space-filling residues. One site corresponding to both a selected cattle ULBP site and a human ULBP3 site that interacts with NKG2D appears in orange.

centrifugation at $10,000 \times g$ for $5 \mathrm{~min}$. The dried pellet was suspended in $40 \mu \mathrm{l}$ of a solution containing $10 \mathrm{mM}$ Tris and $1 \mathrm{mM}$ EDTA.

The BAC DNA was digested using the HindIII restriction enzyme, separated by electrophoresis on 1X TAE agarose, stained using SYBR Green (Invitrogen), and visualized using image analysis (Typhoon Visual Imaging System, Molecular Dynamics) according to an established protocol [15]. Gel images showing restriction fragments were analyzed semiautomatically using IMAGE v3.10b [16], and band migration information was analyzed using FPC v6.0 $[17,18]$ to determine clone overlap for contig assembly as previously described [15].

The first round of sequencing was performed for four minimally overlapping ULBP-containing BACs [GenBank: $\underline{\mathrm{AC} 098687}, \underline{\mathrm{AC} 092858}, \underline{\mathrm{AC} 096629}$ and $\underline{\mathrm{AC} 098686}$ ]. BAC DNA isolation, shotgun cloning, sequencing, quality analysis and sequence assembly were performed using established protocols $[19,20]$. The sequenced BACs were aligned using BLASTN v2.2.13 [21] to generate one contiguous genomic DNA sequence containing three BACs and one singleton. Repetitive elements in the contiguous sequences were masked using REPEATMASKER v3.1.3 [22] before the sequences were used to query publicly available cattle genome trace sequences [NCBI Build 2.0] to identify additional minimally overlapping cattle BACs. Two additional ULBP-containing BAC clones were identified [GenBank: DQ405273 and DQ405274], and a second round of sequencing was performed. Shotgun cloning and sequencing was performed using the Topo Shotgun Subcloning Kit (Invitrogen) according to the manufacturer's instructions. PHRED $[23,24]$ was used to remove low quality sequence (PHRED score < 20). CROSSMATCH and PHRAP [25] were used to remove vector sequence and assemble BAC subclone sequences, respectively. For the two BACs sequenced in the second round, sequence gaps were closed by primer walking. Contiguous sequences [GenBank: DP000081 and DP000082] were constructed from overlapping full-clone BAC sequences using BLASTN. REPEATFINDER [26] was used to identify genomic sequence repeats not identified by REPEATMASKER.

\section{Gene annotation and bioinformatic analysis}

Loci were identified in the repeat-masked contiguous genomic sequences by BLASTN alignment to the GenBank nonredundant [Release 151] and dbEST [Release 012006] databases and by BLASTX alignment to the GenBank nonredundant coding sequence database. The previously identified ULBP1 [GenBank: AF317556] and ULBP2 [GenBank: AY160681] sequences were aligned to the genomic sequences using BLASTN to assist in the annotation of the ULBP genes. Exon/intron boundaries were verified by manual inspection and editing. For each locus identified, all exons were joined and conceptually translated using SIXFRAME [27] to identify open reading frames. Homologous positions in the human genome [NCBI Build 35] were identified using the UCSC Genome Browser [28].

The ULBP multiple alignment was constructed using CLUSTALX [29]. Signal peptides and transmembrane domains were predicted using PSORTII [30], TMPred [31] and TMHMM v2.0 [32]. N-glycosylation and GPI-anchor predictions were carried out using NetNGlyc v1.0 [33] and big-PI predictor [34]. Homology modelling was performed using Swiss-Model and Swiss-PdbViewer [35]. Large-scale alignments were performed for the contiguous sequences using PIPMAKER [36].

\section{Substitution analysis}

Ratios of nonsynonymous to synonymous substitutions $\left(d_{N} / d_{S}\right.$ or $\left.\omega\right)$ were determined for the ULBP genes using the PAML software package [37] to identify evidence of positive Darwinian selection. Cattle sequences used for the substitution analyses included: ULBP1 [GenBank: AF317556], ULBP4 [annotated in GenBank: DP000082], 
ULBP9, ULBP11, ULBP13, ULBP15, ULBP17, ULBP21, ULBP27 and ULBP2 [annotated in GenBank: DP000081] Only the extracellular $\alpha 1$ and $\alpha 2$ domain regions were analyzed. The YN00 program in PAML was used to estimate $\omega_{t}$ for each group of aligned sequences using the method of Yang and Nielsen [38]. The CODEML program in PAML was used to identify variation in selection intensity. The data were modelled using maximum likelihood methods [39], and the results were compared to obtain a test statistic. Three comparisons were performed. Model M1, a neutrality model that constrained $\omega$ to be either 0 or 1 , was compared to both $M 2$, a selection model that added an additional $\omega$ ratio class estimated from the data, and M3, a selection model that used an unconstrained discrete distribution to model classes of $\omega$ ratios. This analysis used three discrete classes for M3. In addition, M7, a continuous distribution neutrality model that estimates $\omega$ using a beta function limited to the interval from 0 to 1 , was compared to $\mathrm{M} 8$, a continuous distribution selection model that adds an additional class of sites with $\omega$ estimated from the data and not constrained to the interval between 0 and 1 . A test statistic of twice the negative value of the difference between the log likelihood values generated under each model was compared to a $\chi^{2}$ distribution with degrees of freedom calculated from the difference in the number of model parameters (M2 vs M1, $\mathrm{df}=2 ; \mathrm{M} 3$ vs $\mathrm{M} 1, \mathrm{df}=4 ; \mathrm{M} 8$ vs $\mathrm{M} 7, \mathrm{df}=2$ ). Posterior probabilities for $U L B P$ sites under positive selection were generated under M8.

\section{Authors' contributions}

JHL carried out sequence analysis and annotation, substitution analysis, homology modelling, interpretation of data, participated in the design of the study, and drafted the manuscript. BMM carried out BAC clone fingerprinting and selection of minimally overlapping clones. JEB performed sequence analysis and assembly and participated in the design of the study. BAR contributed BAC sequences. HAL supervised the research, participated in the design of the study, interpretation of data, and drafting of the manuscript. All authors have read the manuscript, provided critical reviews of content, and approved the final manuscript.

\section{Acknowledgements}

The authors thank: Denis Larkin at the University of Illinois, Urbana-Champaign, Yongjou Yoon, Steve Shaull, and Ziyun Yao of the Advanced Center for Genome Technology at the University of Oklahoma, and Alvaro Hernandez of the W.M. Keck Center for Comparative and Functional Genomics at the University of Illinois at Urbana-Champaign for technical advice and assistance with BAC sequencing. This study and the authors' contributions to it were funded by a grant to HAL from the USDA National Research Initiative (AG2002-35205- I I 625) and a grant to BAR from NIH NHGRI (HG02I52). These funding bodies played no role in the design, collection, analysis, interpretation, writing, or the decision to submit the manuscript for publication.

\section{References}

I. Larson JH, Rebeiz MJ, Stiening CM, Windish RL, Beever JE, Lewin HA MHC class I-like genes in cattle, MHCLA, with similarity to genes encoding NK cell stimulatory ligands. Immunogenetics 2003, 55:16-22.

2. Cosman D, Müllberg J, Sutherland CL, Chin W, Armitage R, Fanslow W, Kubin M, Chalupny NJ: ULBPs, novel MHC class I-related molecules, bind to CMV glycoprotein ULI6 and stimulate NK cytotoxicity through the NKG2D receptor. Immunity 200 I, 14:123-133.

3. Radosavljevic M, Cuillerier B, Wilson MJ, Clément O, Wicker S, Gilfillan S, Beck S, Trowsdale J, Bahram S: A cluster of ten novel MHC class I related genes on human chromosome 6q24.2-q25.3. Genomics 2002, 79: I | 4- 123.

4. Nomura M, Takihara $Y$, Shimada K: Isolation and characterization of retinoic acid-inducible cDNA clones in F9 cells: one of the early inducible clones encodes a novel protein sharing several highly homologous regions with a Drosophila polyhomeotic protein. Differentiation 1994, 57:39-50.

5. Zou Z, Nomura M, Takihara Y, Yasunaga T, Shimada K: Isolation and characterization of retinoic acid-inducible cDNA clones in F9 cells: a novel cDNA family encodes cell surface proteins sharing partial homology with MHC class I molecules. J Biochem (Tokyo) 1996, I I 9:319-328.

6. Malarkannan S, Shih PP, Eden PA, Horng T, Zuberi AR, Christianson $G$, Roopenian D, Shastri N: The molecular and functional characterization of a dominant minor $\mathbf{H}$ antigen, $\mathbf{H 6 0}$. I Immunol 1998, I61:3501-3509.

7. Carayannopoulos LN, Naidenko OV, Fremont DH, Yokoyama WM: Costimulation through NKG2D enhances murine CD8+ CTL function: similarities and differences between NKG2D and CD28 costimulation. J Immunol 2005, I 75:2825-2833.

8. Bahram S, Inoko H, Shiina T, Radosavljevic M: MIC and other NKG2D ligands: from none to too many. Curr Opin Immunol 2005, 17:505-509.

9. Kubin M, Cassiano L, Chalupny J, Chin W, Cosman D, Fanslow W, Müllberg J, Rousseau AM, Ulrich D, Armitage R: ULBP I, 2, 3: novel MHC class I-related molecules that bind to human cytomegalovirus glycoprotein UL I6, activate NK cells. Eur J Immunol 2001, 3 I : | 428-| 437.

10. Dunn C, Chalupny NJ, Sutherland CL, Dosch S, Sivakumar PV, Johnson DC, Cosman D: Human cytomegalovirus glycoprotein UL 16 causes intracellular sequestration of NKG2D ligands, protecting against natural killer cell cytotoxicity. J Exp Med 2003, 197:|427-1439.

II. García-Borges CN, Phanavanh B, Saraswati S, Dennis RA, Crew MD: Molecular cloning and characterization of a porcine ULI6 binding protein (ULBP)-like cDNA. Mol Immunol 2005 , 42:665-67l.

12. Nei M, Gojobori T: Simple methods for estimating the numbers of synonymous and nonsynonymous nucleotide substitutions. Mol Biol Evol 1986, 3:418-426.

13. Endo T, Ikeo K, Gojobori T: Large-scale search for genes on which positive selection may operate. Mol Biol Evol 1996 , I 3:685-690.

14. Radaev S, Rostro B, Brooks AG, Colonna M, Sun PD: Conformational plasticity revealed by the cocrystal structure of NKG2D and its class I MHC-like ligand ULBP3. Immunity 200 I, I 5:1039-1049.

15. Marra MA, Kucaba TA, Dietrich NL, Green ED, Brownstein B, Wilson RK, McDonald KM, Hillier LW, McPherson JD, Waterston RH: High throughput fingerprint analysis of large-insert clones. Genome Res 1997, 7:1072-1084.

16. Sulston J, Mallett F, Durbin R, Horsnell T: Image analysis of restriction enzyme fingerprint autoradiograms. Bioinformatics 1989, 5:101-106.

17. Soderlund C, Longden I, Mott R: FPC: A system for building contigs from restriction fingerprinted clones. CABIOS 1997 , 1 3:523-535.

18. Soderlund C, Humphray S, Dunham A, French L: Contigs built with fingerprints, markers, and FPC V4.7. Genome Res 2000, I 0:1772-1787.

19. Roe BA, Crabtree JS, Khan AS: DNA Isolation and Sequencing Hoboken: John Wiley and Sons; 1996.

20. Protocols used in the Roe Laboratory [http:// www.genome.ou.edu/proto.html] 
21. Altschul SF, Madden TL, Schaffer AA, Zhang J, Zhang Z, Miller W, Lipman DJ: Gapped BLAST and PSI-BLAST: a new generation of protein database search programs. Nucleic Acids Res 1997, 25:3389-3402.

22. Repeatmasker [http://www.repeatmasker.org]

23. Ewing B, Hillier L, WendI MC, Green P: Base-calling of automated sequencer traces using phred. I. Accuracy assessment. Genome Res 1998, 8: 175-185.

24. Ewing B, Green P: Base-calling of automated sequencer traces using phred. II. Error probabilities. Genome Res 1998, 8:186-194.

25. PHRED, PHRAP, CONSED [http://www.phrap.org/phredphrap consed.html]

26. Volfovsky N, Haas BJ, Salzberg SL: A clustering method for repeat analysis in DNA sequences. Genome Biol 200I, 2:RESEARCH0027.I-0027.II.

27. Biology Workbench [http://workbench.sdsc.edu/]

28. Kent W], Sugnet CW, Furey TS, Roskin KM, Pringle TH, Zahler AM, Haussler D: The human genome browser at UCSC. Genome Res 2002, I 2:996-1006.

29. Jeanmougin F, Thompson JD, Gouy M, Higgins DG, Gibson TJ: Multiple sequence alignment with Clustal $X$. Trends Biochem Sci 1998, 23:403-405.

30. Nakai K, Kanehisa M: A knowledge base for predicting protein localization sites in eukaryotic cells. Genomics 1992, |4:897-9||.

31. Hofmann K, Stoffel W: Tmbase - a database of membrane spanning protein segments. Biol Chem Hoppe-Seyler 1993, 374:166.

32. TMHMM Server v. 2.0 [http://www.cbs.dtu.dk/services/TMHMM2.0/]

33. NetNGlyc I.0 Server [http://www.cbs.dtu.dk/services/NetNGlycl ]

34. Eisenhaber B, Bork P, Eisenhaber F: Prediction of potential GPImodification sites in proprotein sequences. J Mol Biol 1999, 292:74I-758.

35. Guex N, Peitsch MC: SWISS-MODEL and the Swiss-PdbViewer an environment for comparative protein modeling. Electrophoresis 1997, 18:27|4-2723.

36. Schwartz S, Zhang Z, Frazer KA, Smit A, Riemer C, Bouck J, Gibbs R, Hardison R, Miller W: PipMaker-A Web Server for Aligning Two Genomic DNA Sequences. Genome Res 2000, 10:577-586.

37. Yang Z: PAML: a program package for phylogenetic analysis by maximum likelihood. Comput Appl Biosci 1997, 13:555-556.

38. Yang Z, Nielsen R: Estimating synonymous and nonsynonymous substitution rates under realistic evolutionary models. Mol Biol Evol 2000, 17:32-43.

39. Yang Z, Nielsen R, Goldman N, Pedersen AM: Codon-substitution models for heterogeneous selection pressure at amino acid sites. Genetics 2000, I55:43 I-449.

Publish with Bio Med Central and every scientist can read your work free of charge

"BioMed Central will be the most significant development for disseminating the results of biomedical research in our lifetime. "

Sir Paul Nurse, Cancer Research UK

Your research papers will be:

- available free of charge to the entire biomedical community

- peer reviewed and published immediately upon acceptance

- cited in PubMed and archived on PubMed Central

- yours - you keep the copyright
BioMedcentral 